\title{
Design and Realization of Stored-Value Payment System
}

\author{
JIANG Ning , ZHU- Xiao Dong \\ Information School, Ningbo City College of Vocational Technology,ningbo,China \\ Information School, Ningbo City College of Vocational Technology,ningbo,China \\ 48971892@qq.com , zhuxiaodong@nbcc.cn \\ (Zhejiang Provincial Education Department Project No: ZWT15144)
}

Keywords: deposit and payment; Common Object Request Broker Architecture(CORBA); isomerism systems;TUXEDO components

\begin{abstract}
This paper intends to study and build deposit and payment platform System ,According to the feature of business requirements, The system has been designed on the basis of CORBA midware, Because the system needs to interact with the legacy systems of the telecom with which having different program languages and different databases, To integrate these isomerism systems ,Finally adopt CORBA infrastructure to encapsulate the system ;Utilized the TUXEDO components as application server ; and proposed five vital design schemas and have come to fulfillment. And finally established a deposit and payment platform with Advanced architecture.
\end{abstract}

\section{Foreword}

Currently, most e-commerce sites use their own certification system, the user must be registered in different sites can use one of the service, and the user name and password are likely not the same, remember different user name and password for every site for non-professional users, it is very difficult. There is an urgent need for a unified certification system ${ }^{[1]}$, so "card in the world", and application service providers also need such a system in line with China's national conditions to meet the needs of users.

Another on the Internet so that the user is inconvenienced payment issues. Currently, there are three methods of payment: cash on delivery, card payment bank, post office remittances, according to CNNIC survey showed that the bank card payment only to $1 / 3$. Now that the bank card payment is still fragmented, different interfaces, different mode of operation, the different card different passwords, all gave users a barrier use. Similarly, people need a unified payment system, simplify people's operation; and application service providers also need a unified access system to achieve access to the respective banks.

Stored value payment platform is proposed in this context, to unified authentication, unified payment, unified access to three characteristics, to meet the needs of users and ASP.

Stored value card payment platform to achieve lifelong unique one associated with the user identity to a variety of security measures to protect the security of user information; a unified interface and access each bank, to provide a unified method of payment; to access services provide third-party service, keeping the platform scalability and flexibility. Multi Account Manager enables users to have a more flexible funding management; relationship management allows the user with a different bank cards, different third-party service relationship binding enjoy more services. Stored value payment platform also provides integration, promotions and other modern marketing methods, and can be different kinds of query statistics as required.

The system is hosted by our design and development platform is based on CORBA architecture ${ }^{[2]}$, the use of middleware (CORBA Server) Tuxedo 8.0 technology, the eventual establishment of a set of advanced infrastructure, improve the function of the stored value payment platform.

System Architecture Design. Architecture stored-value payment platform as shown below, 
stored-value payment platform is based on a business Corba middleware middleware products.

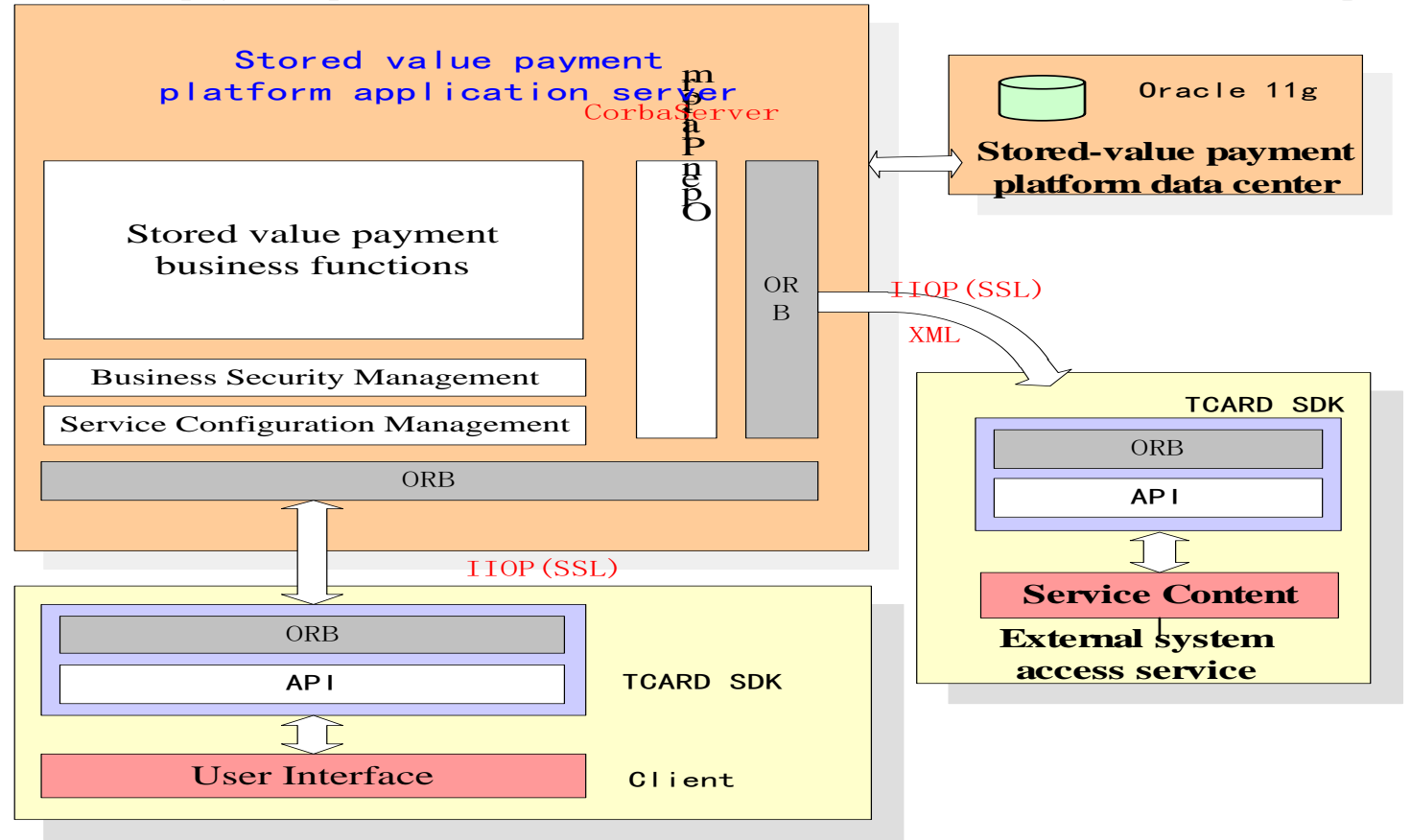

Fig.1 Stored value payment platform architecture design

Stored value payment platform core.Application Server is the core of stored-value payment platform, responsible for all business logic processing. Stored value payment platform application server is based on CORBA middleware ${ }^{[3]}$ The product supports multiple OS platforms: including win2000 / win7, Linux and a variety of Unix platforms.

Stored value payment card core business functions as customer relationship management, account relationship management, business relationship management, benefits and integration, data auditing, statistical analysis; system configuration and security management is the foundation of the entire platform system, rely on this module, the platform each module provides flexible configuration management and strong security; open interfaces provide open business platform for stored value payment.

Responsible for the business logic of the application processing server. Including protocol software application server management software, business software, and business processing gateway. And connect all the systems which are by Corba standard protocol IIOP.

Management Environment.Stored value payment platform has name species management and monitoring tools, you can keep abreast of prepaid payment platform application server and the various components of the current operating status, such as the current processing application server load operating conditions of the various access systems, connection status data, remote server connection status and the like. These functions can be when the stored value payment platform fails to quickly find the source of failure to provide effective help.

Payment platform business functions

Stored value payment platform system is based on customer base; customer funds account management as the core; to provide authentication, fund management, payment services, national roaming, one card can function; the company offers marketing, customer management means, business analysis, information gathering, etc. Here is a brief description of each function module.

User management and customer relationship management.User information management platform stored value payment system, including query the user resource management, user authentication, managing the relationship between the card and user information. Only users authenticated stored value payment platform, only You can use and apply key business in the platform.

System fully into account the enterprises of various types of customers, such as large customers, small and medium customers, designed for families and individuals of personal cards, 
business-oriented enterprises and departments of the main card, corporate daughter card.

\section{Key technical design}

Deployment architecture based on multiple layers of middleware.For a platform, scalability and openness are very important.Stored value payment platform system is based on an open platform CORBA, stored value payment services to provide some expansion capability, flexibility and scalability.The entire platform system is divided into four layers: data storage layer, the object management, business framework component layer, service access layer. Application of the model in line with the hierarchical, component standardization, to meet the distribution, heterogeneous, dynamic and open nature of complex business Stored value payment platform:

Extended deployment based on the number of users to upgrade the system;

Expansion of business strategies based on changes in the business;

Middleware ${ }^{[4]}$ Without limiting characteristics in combination with external systems and external applications.

Rapid Development.Stored value payment platform system application interface based on CORBA standard gateway unified interface. And business application systems support a variety of connection hardware platforms and operating systems, application system access provides a consistent application programming interface that allows applications to use any language that supports CORBA software developers, such as C, C ++, Java, C \# and Small talk, etc. to write the interface, not the operating system limitation, in line with the company's existing software architecture development scale capabilities to meet the rapid construction of the integrated business and flexible deployment.

At the same time, based on CORBA technology, you can easily achieve portability systems, interoperability, and distribution transparency, easily expand and upgrade the system.

\section{Principle platform.}

We follow a few design concepts developed above a SDK development kits, development kits can be simplified by the development of prepaid payment platform client and access system.

Open Interface. Open Interface as a bridge between the stored value payment platform and access servers, so that access system to access a stored value payment platform services (access services via ServiceCenter interface. ${ }^{[7]}$ to access the stored value payment platform services), while prepaid payment service platform access access services provided by the system (stored-value payment services platform provided by PluginServer interface to access the access server). Each access service system must be certified payment platform refill, before you can call the appropriate service stored value payment platform, each access service system must be registered to a stored value payment platform in order to provide services to the prepaid payment platform.

Access Services. Access is available CORBA interface (Plugin Server) for prepaid payment platform invoke Tuxedo ${ }^{[8]}$ service, in essence, an access server is a CORBA server, we offer access services API, so developers do not have access to care and services CORBA problem.

\section{Achieve the effect of the system}

After the stored value payment platform system construction is completed, in order to achieve the effect of verification platform, we mainly test and other services for the correctness of logic systems and pressures for testing.

System Services business logic test. Test is initiated by the client corba call corba service, then by corba service call pluginServer, then by pluginServer into external systems to communicate.

Test Preparation: registered access system, commercial banks registered in the CORBA server 11620 to go. The main contents of the tests as described in the following table: 
Table 1 Access services logic test table

\begin{tabular}{|l|c|}
\hline \multicolumn{1}{|c|}{ Logical Test } & In conclusion \\
\hline Test transferFrom service (by the bank to service card) & Normal service \\
\hline Test rollback Trade Service & Normal service \\
\hline Test unbinduser Service (lift service card and bank account bindings) & Normal service \\
\hline Test bindUser service (this service relates to the encryption algorithm) & Normal service \\
\hline
\end{tabular}

System Services Stress Test. Stress testing using LoadRunner stress testing tool to simulate 10 000 online debit transactions, call interface (UA_transfer) Time spent four minutes and eight seconds; simulated 10,000 online inquiries transaction, call interface (SA_call), the time it took 4 minutes zero 42 seconds, the two major trade pressure test time is less than the system maximum pressure threshold time (five minutes), so the concurrent performance of the platform services is very good.

\section{Conclusion}

The stored-value payment platform currently running well, but we think we need to make further improvements in the following areas:

currently stored value payment platform is still relatively loose management rights in the future need to use a unified, centralized manner.

Exception error when executing the definition is not precise enough, does not call link information. Ability platform also currently no handle large integer and floating point, Long type in some businesses have been unable to meet the requirements.

\section{References}

[1] Xuguanhua. The importance of a unified certification system. Computer, 2013, (1): 25-32

[2] Peng Muhuo. CORBA Technology and Realization. Beijing: Electronic Industry Press, 2010, $10-15$

[3] W.H.Inmon. Building the CORBA architecture (3rd Edition). John Wiley\& Sons, 57Inc. 2011, 21-30

[4] Trujillo J, Palomar M.GomezJ.Song I. Designing Middle Ware Components with OOConceptual Models, IEEE Computer, 2012，34(12): 66-75

[5] CHEN Chongcheng. Multi-tier application architecture [Design and Implementation. Southeast University, 2010, 32 (05): 163-170

[6] S.Chaudhuri.U.Dayal • An Overview of XML Technology, SIGMOD Record, 2010, (26): 23-28

[7] Li Liming. Service Interface implementation of key technologies, Computer, 2012, (3): 30-34

[8] R.Agrawal, R.Srikant. . Building the Tuxedo Components. In Proc. 20th VLDB, 2012, (9): 487-499 